\title{
A Local Theory of Entangled Photons That Matches QM Predictions
}

\author{
Richard A. Hutchin \\ Optical Physics Company, Calabasas, USA \\ Email: rahutchin@opci.com
}

Received 30 July 2014; revised 29 August 2014; accepted 21 September 2014

Copyright (C) 2014 by author and Scientific Research Publishing Inc.

This work is licensed under the Creative Commons Attribution International License (CC BY). http://creativecommons.org/licenses/by/4.0/

c) (i) Open Access

\begin{abstract}
Bell's theorem, first presented by John Bell in 1964, has been used for many years to prove that no classical theory can ever match verified quantum mechanical predictions for entangled particles. By relaxing the definition of entangled slightly, we have found a mathematical solution for two entangled photons that produces the familiar quantum mechanical counting statistics without requiring a non-local theory such as quantum mechanics. This solution neither is claimed to be unique nor represents an accurate model of photonic interactions. However, it is an existence proof that there are local models of photonic emission that can reproduce quantum statistics.
\end{abstract}

\section{Keywords}

Entangled Photons, Bell's Theorem, Local Theory of Light

\section{Introduction}

One of the strongest constraints on a local theory of light is Bell's theorem [1]. Bell's theorem is usually interpreted to mean that any local model of physics cannot satisfy the type of counting statistics predicted by quantum mechanics. Since these statistics have been verified fairly well, the conclusion is that there is no possible local theory of physics where all interactions are determined by the local properties of fields.

The logic of Bell's theorem proceeds as follows: any local theory of light says that for a given E\&M pulse from a photonic decay, one can write the probability of detection as a function of polarizer angle $\theta$ by a simple function $P_{0}(\theta, a)$ where $a$ is an unspecified set of hidden variables identifying the state of the photonic pulse. As illustrated in Figure 1, for two entangled photons, the probability that one will be detected in polarization state $\theta_{1}$ and the second in polarization state $\theta_{2}$ is:

$$
P_{12}\left(\theta_{1}, \theta_{2}, a\right)=P_{0}\left(\theta_{1}, a\right) \cdot P_{0}\left(\theta_{2}, a\right)
$$




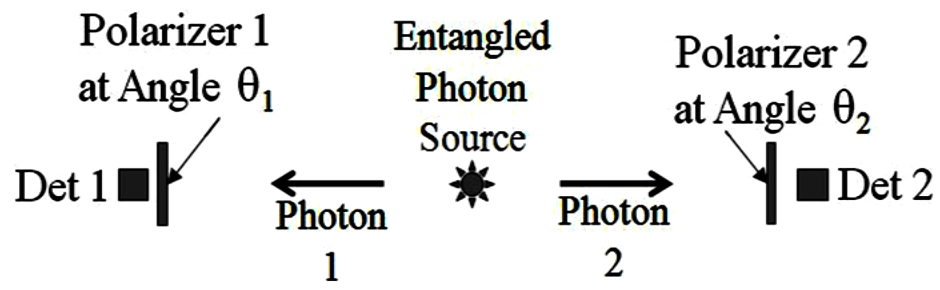

Figure 1. The Bell's theorem test of quantum mechanics uses a source of entangled photons, where each photon goes to a different detector through two different polarizers. The probability of simultaneous detection is proportional to $\cos \left(\theta_{1}-\theta_{2}\right)^{2}$.

If we assume from rotational symmetry of the source that one of the hidden parameters is an angle $\phi$ (such as the polarization direction of the emitted photon), which rotates the probability function uniformly over the polarizer angle in the detector, then we can write Equation (1) as shown below in Equation (2) where $a^{\prime}$ is the rest of the hidden variables which are assumed to be independent of $\phi$.

$$
P_{12}\left(\theta_{1}, \theta_{2}, \phi, a^{\prime}\right)=P_{0}\left(\theta_{1}-\phi, a^{\prime}\right) \cdot P_{0}\left(\theta_{2}-\phi, a^{\prime}\right)
$$

By averaging over $\phi$, we get Equation (3) which can be rewritten in the form of a convolution by changing variables as shown in Equation (4). (Replace $\theta_{2}-\phi$ by $\phi^{\prime}$.)

$$
\begin{aligned}
& P_{12}\left(\theta_{1}, \theta_{2}, a^{\prime}\right)=\frac{1}{2 \pi} \cdot \int_{0}^{2 \pi} P_{0}\left(\theta_{1}-\phi, a^{\prime}\right) \cdot P_{0}\left(\theta_{2}-\phi, a^{\prime}\right) \mathrm{d} \phi \\
& P_{12}\left(\theta_{1}, \theta_{2}, a^{\prime}\right)=\frac{1}{2 \pi} \cdot \int_{0}^{2 \pi} P_{0}\left(\theta_{1}-\theta_{2}+\phi^{\prime}, a^{\prime}\right) \cdot P_{0}\left(\phi^{\prime}, a^{\prime}\right) \mathrm{d} \phi^{\prime}
\end{aligned}
$$

We can now average over the other potential hidden variables $a^{\prime}$ to get the final result in Equation (5).

$$
P_{12}\left(\theta_{1}, \theta_{2}\right)=\operatorname{Mean}_{a^{\prime}}\left(\frac{1}{2 \pi} \cdot \int_{0}^{2 \pi} P_{0}\left(\theta_{1}-\theta_{2}+\phi^{\prime}, a^{\prime}\right) \cdot P_{0}\left(\phi^{\prime}, a^{\prime}\right) \mathrm{d} \phi^{\prime}\right)
$$

Quantum mechanics says that the result must be Equation (6).

$$
P_{12}\left(\theta_{1}, \theta_{2}\right)=\frac{1}{\pi} \cdot \cos \left(\theta_{1}-\theta_{2}\right)^{2}
$$

Bell's theorem says that there is no function $P_{0}\left(\phi^{\prime}, a^{\prime}\right)$ for any possible set of hidden variables $a^{\prime}$ which can make Equation (5) match Equation (6) —not even close. The simple mathematical principle which leads to this conclusion is that there is no non-negative real function which can be correlated with itself to give $\cos (\theta)^{2}$. This principle applies here because $P_{0}$ is a probability function and must be nonnegative. The short proof is summarized below.

\section{Short Proof of Bell's Theorem for the Given Formulation}

The easy way to prove that there can be no solution $P_{0}$ to Equation (5) which matches Equation (6) is to write both Equation (5) and Equation (6) using Fourier components. Equation (7) shows a general probability function $P_{0}$ written in terms of its Fourier components $P F_{0}$.

$$
P_{0}(\theta)=\frac{1}{2 \pi} \sum_{k=-\infty}^{\infty}\left(P F_{0_{k}} \mathrm{e}^{i \cdot k \cdot \theta}\right)
$$

Equation (8) shows the familiar result that when $P_{0}$ is convolved with itself, as in Equation (5), the Fourier components of the result equal the magnitude-squared of the Fourier components of $P_{0}$.

$$
P_{12}\left(\theta_{1}, \theta_{2}\right)=\frac{1}{2 \pi} \sum_{k=-\infty}^{\infty}\left[\left(\left|P F_{0_{k}}\right|\right)^{2} \cdot \mathrm{e}^{i \cdot k \cdot\left(\theta_{1}-\theta_{2}\right)}\right]
$$


Now experimental data require that the answer must be Equation (9).

$$
\begin{aligned}
P_{12}\left(\theta_{1}, \theta_{2}\right) & =\frac{1}{\pi} \cdot \cos \left(\theta_{1}-\theta_{2}\right)^{2} \\
& =\frac{1}{2 \pi} \cdot\left[\frac{1}{2} \cdot \mathrm{e}^{-2 \cdot i \cdot\left(\theta_{1}-\theta_{2}\right)}+1+\frac{1}{2} \cdot \mathrm{e}^{2 \cdot i \cdot\left(\theta_{1}-\theta_{2}\right)}\right]
\end{aligned}
$$

Since the Fourier components of $P_{12}$ are the magnitude-squared of the Fourier components of $P_{0}$, we see that the magnitudes of the three Fourier components for $P_{0}$ have to be

$$
\left|P F_{0_{-2}}\right|=\frac{1}{\sqrt{2}} \quad\left|P F_{0_{0}}\right|=1 \quad\left|P F_{0_{2}}\right|=\frac{1}{\sqrt{2}}
$$

Equation (10) does not tell us what the phase is for the second Fourier components, only the magnitude, so if we give the phase a name $\varphi$, and note that the two Fourier components have to be complex conjugates of each other to make a real probability function, we get the most general form for the probability distribution $P_{0}$ which can convolve to give the experimentally verified result in Equation (9).

$$
\begin{aligned}
P_{0}(\theta) & =\frac{1}{2 \pi}\left(\frac{1}{\sqrt{2}} \mathrm{e}^{-2 \cdot \cdot \cdot \theta-\varphi}+1+\frac{1}{\sqrt{2}} \mathrm{e}^{2 \cdot i \cdot \theta-\varphi}\right) \\
& =\frac{1}{2 \pi}\left(1+\frac{1}{\sqrt{2}} \cos (2 \theta+\varphi)\right)
\end{aligned}
$$

The minimum of Equation (11) is -0.414 , which violates the principle that a probability function must be non-negative. This is why it is said that no classical theory can even come close to the quantum mechanical result-providing a strong argument in favor of quantum mechanics.

\section{Rethinking Bell's Theorem}

Bell's theorem is a mathematic result which follows from its assumptions. To construct a successful local theory, we need to loosen one of the assumptions which led to the contradiction. One subtle but critical assumption is that the set of hidden variables $a^{\prime}$ applies identically to both E\&M pulses (i.e., both of the entangled photons). This assumption makes each of the probability functions identical. We suggest alternatively that what is required instead is that the two photons be tied together in a symmetric manner so that, over the allowed range of hidden variables, they cannot be distinguished in their properties. This change in the definition of an entangled state allows us to match experimental results with a local theory.

In this simplest implementation of this altered model of entangled photons, the pair of photons have different probability functions versus polarization angle, $P_{1}(\theta)$ and $P_{2}(\theta)$. Assignment of a distribution to a particular one of the two photons is random so that statistically the two photons are identical. Even more if one entangled photon has probability distribution $P_{1}$ then the other photon has probability distribution $P_{2}$ and vice versa. Thus the two photons are tightly locked together.

With this formulation, the next step is to choose two different real, non-negative functions over 0 - $2 \pi$ which integrate to unity and whose cross-correlation probability distribution is $\cos (\theta)^{2} / \pi$ Obviously there is considerably more freedom to find solutions here. Written in equation form, the constraints on $P_{1}$ and $P_{2}$ are given by Equations (12).

$$
\begin{aligned}
& P_{1}(\theta) \geq 0 \quad P_{2}(\theta) \geq 0, \\
& \int_{0}^{2 \pi} P_{1}(\theta) \mathrm{d} \theta=\int_{0}^{2 \pi} P_{2}(\theta) \mathrm{d} \theta=1, \\
& \int_{0}^{2 \pi} P_{1}\left(\theta-\theta_{1}\right) \cdot P_{2}\left(\theta-\theta_{2}\right) \mathrm{d} \theta=\frac{1}{\pi} \cdot \cos \left(\theta_{1}-\theta_{2}\right)^{2},
\end{aligned}
$$

The only solution we have found where each function is finitely differentiable in the nonzero quadrants is given in Equations (13) and plotted in Figure 2. Note that both functions are nonzero only over half the $0-2 \pi$ domain. This is required to get zero correlation at a 90 degree angular difference. The two functions are plotted versus angle in Figure 2. 


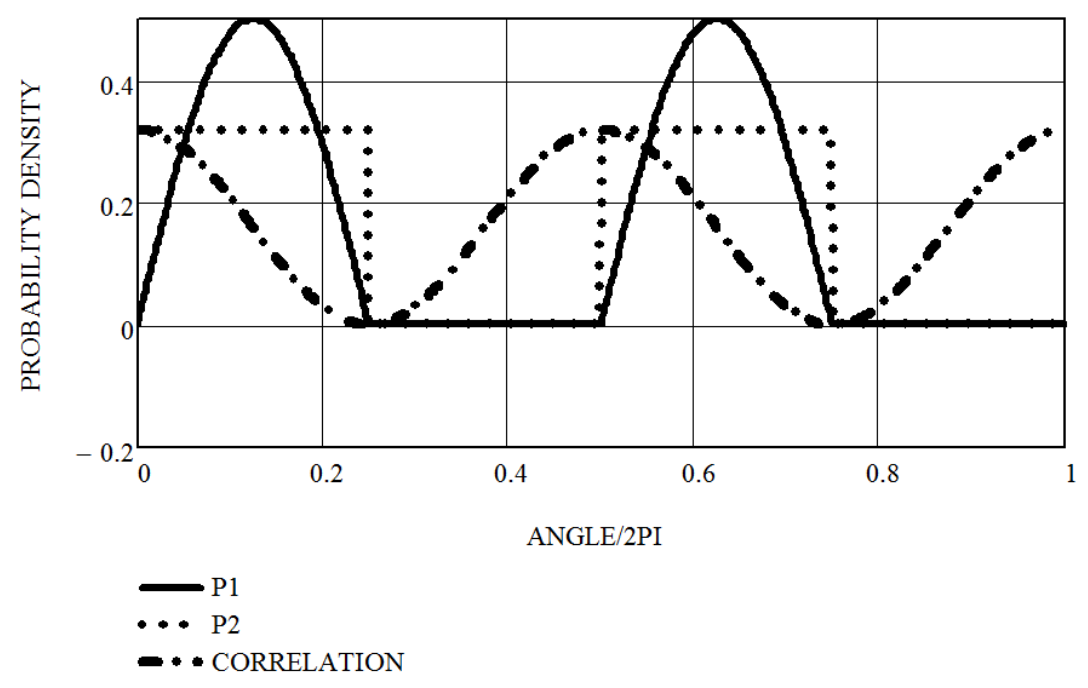

Figure 2. Plot of two functions which cross-correlate to give the experimental $\cos (\theta)^{2} / \pi$ polarization correlation.

$$
\begin{aligned}
& P_{1}(\theta)= \begin{cases}\frac{\sin (2 \theta)}{2} & \text { if } \sin (2 \theta)>0 \\
0 & \text { otherwise }\end{cases} \\
& P_{2}(\theta)= \begin{cases}\frac{1}{\pi} & \text { if } \bmod (\theta, \pi) \leq \frac{\pi}{2} \\
0 & \text { otherwise }\end{cases}
\end{aligned}
$$

\section{Discussion}

Bell's theorem has long been considered a fundamental barrier to classical theories of light interaction with matter. C.B. Parker [2] summed up Bell's theorem by saying, "No physical theory of local hidden variables can ever reproduce all of the predictions of quantum mechanics.” And Henry Stapp [3] summarized a broad feeling among scientists that: "Bell's theorem is the most profound discovery of science."

The original test of Bell's theorem used entangled electrons, but soon after that entangled photons were the experimental choice. John Clauser and Stuart Freedman [4] and later Alain Aspect et al. [5] demonstrated that the predictions of QM were accurately matched by experimental data. Clauser and Freedman summarized their work below:

"We have measured the linear polarization correlation of the photons emitted in an atomic cascade of calcium. It has been shown by a generalization of Bell's inequality that the existence of local hidden variables imposes restrictions on this correlation in conflict with the predictions of quantum mechanics. Our data, in agreement with quantum mechanics, violate these restrictions to high statistical accuracy, thus providing strong evidence against local hidden-variable theories.”

Quantum mechanics requires the collapse of a probability wave as one of the photons passes through its polarizer, which then determines - faster than the speed of light-whether the other photon would pass through its polarizer. This connection has been probed in many forms over the last half century since Bell's seminal 1964 paper [1].

A 2014 paper called Bell Nonlocality by Bruner, N. et al. [6] comprehensively summarizes research into Bells' theorem especially in regard to its nonlocal behavior with over 500 references. This nonlocal behavior has led to a difficult concept where what happens to one photon will determine what happens to the other even if the two are far away. Even stranger, this nonlocal interaction must happen faster than the speed of light to prevent one 
photon from being in a measured state inconsistent with the other.

The key advantage of a local theory of light is that this mysterious process that communicates the collapse of a quantum state faster than the speed of light is no longer required. The two photons agree with each other because they are in deterministic interlocked states. What we have found here is that by allowing the entangled photons to have independent but fixed probabilities of detection with the angle of their polarizers, there is indeed a local model of interaction that matches the predictions of quantum mechanics.

The probability functions that result are strange looking to us and not explained or predicted by current theories of light, and yet the experimentally verified zero correlation of counts when the two polarizations are set $90^{\circ}$ apart forces the two functions to have $90^{\circ}$ sections with zero probability of detection. If there is a local theory of light interaction, it will have to have probability curves similar to those shown here.

\section{Conclusions}

One might conclude that such behavior of detection probability with angle would violate experimental data, but no one has ever measured the probability distribution of a single entangled photon. No one has even proposed a technique to do that. The reason why it is so difficult is that the orientation of a random pair of photons is random and thus always has an equal probability of being detected at any angle of a polarizer. We can relate the probability of one photon detection correlating with the other, but so far we have no way to measure one photon's probability of detection versus angle by itself.

This paper has shown there are probability functions for individual entangled photons that will match verified quantum mechanical predictions. While many questions come up here, such as what theory of light would predict such probability of detection functions, we suggest that Bell's theorem as a mathematical result should no longer be considered a definitive proof of non-local interaction.

\section{References}

[1] Bell, S. (1964) On the Einstein Podolsky Rosen Paradox. Physics, 1, 195-200.

[2] Parker, C.B. (1994) McGraw-Hill Encyclopedia of Physics. 2nd Edition, McGraw-Hill, New York, 542.

[3] Stapp, H.P. (1975) Bell's Theorem and World Process. Nuovo Cimento, 29B, 270. http://dx.doi.org/10.1007/BF02728310

[4] Freedman, S. and Clauser, J. (1972) Experimental Test of Local Hidden Variable Theories. Physical Review Letters, 28, 938. http://dx.doi.org/10.1103/PhysRevLett.28.938

[5] Aspect, A., et al. (1981) Experimental Tests of Realistic Local Theories via Bell’s Theorem. Physical Review Letters, 47, 460. http://dx.doi.org/10.1103/PhysRevLett.47.460

[6] Bruner, N., et al. (2014) Bell Nonlocality. Reviews of Modern Physics, 86, 419.

http://dx.doi.org/10.1103/RevModPhys.86.419 
Scientific Research Publishing (SCIRP) is one of the largest Open Access journal publishers. It is currently publishing more than 200 open access, online, peer-reviewed journals covering a wide range of academic disciplines. SCIRP serves the worldwide academic communities and contributes to the progress and application of science with its publication.

Other selected journals from SCIRP are listed as below. Submit your manuscript to us via either submit@scirp.org or Online Submission Portal.
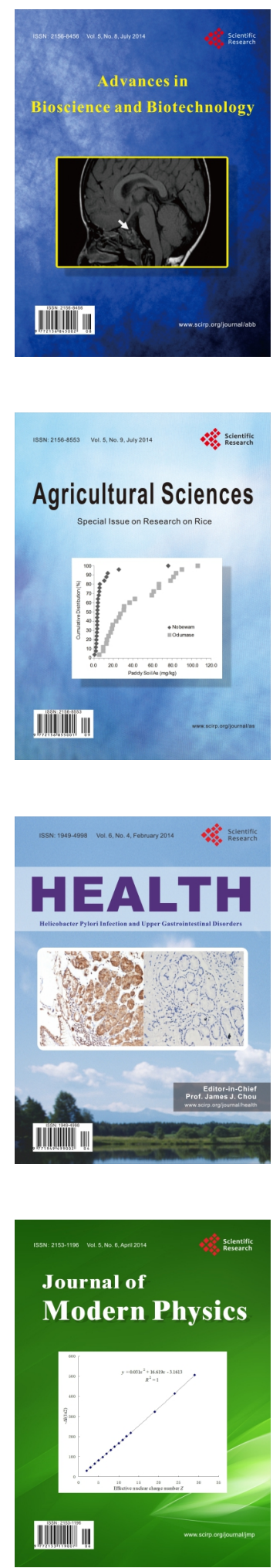
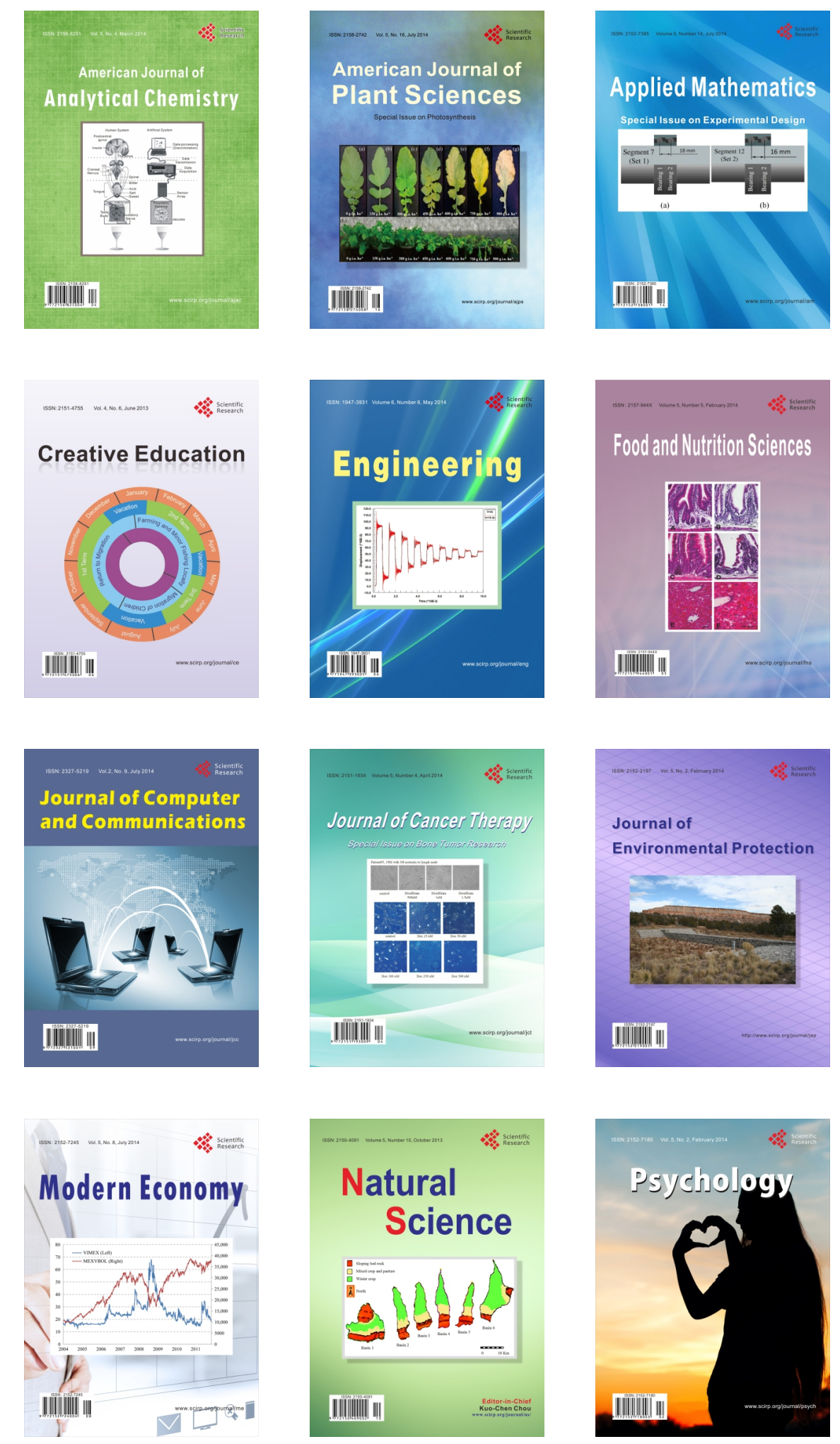